\title{
Design of A high performance low-power consumption discrete time Second order Sigma-Delta modulator used for Analog to Digital Converter
}

\author{
Radwene LAAJIMI \\ University of Sfax \\ Electronics, Micro-technology and Communication \\ (EMC) research group \\ Sfax (ENIS), BP W, 3038 Sfax, Tunisia
}

\author{
Mohamed MASMOUDI \\ University of Sfax \\ Electronics, Micro-technology and Communication \\ (EMC) research group \\ Sfax (ENIS), BP W, 3038 Sfax, Tunisia
}

\begin{abstract}
This paper presents the design and simulations results of a switched-capacitor discrete time Second order Sigma-Delta modulator used for a resolution of 14 bits Sigma-Delta analog to digital converter. The use of operational amplifier is necessary for low power consumption, it is designed to provide large bandwidth and moderate DC gain. With $0.35 \mu \mathrm{m}$ CMOS technology, the $\Sigma \Delta$ modulator achieves $86 \mathrm{~dB}$ dynamic range, and $85 \mathrm{~dB}$ signal to noise ratio (SNR) over an $80 \mathrm{KHz}$ signal bandwidth with an oversampling ratio (OSR) of 88 , while dissipating $9.8 \mathrm{~mW}$ at $\pm 1.5 \mathrm{~V}$ supply voltage.
\end{abstract}

Keywords- CMOS technology; Analog-to-Digital conversion; Low power electronics; Sigma-Delta modulation; switchedcapacitor circuits; transconductance operational amplifier.

\section{INTRODUCTION}

Analog to digital converters (ADC) with high resolution are widely used in the areas of instrumentation, measurement, telecommunications, digital signal processing, consumer electronics, etc. With the advancements in the Very Large Scale Integration (VLSI) technologies, the focus is shifted on oversampling and $\Delta \Sigma$ converters for applications requiring high precision analog-to digital conversion with narrow bandwidth $[1,2,3,4]$. They are preferred because of their inherent relaxed sensitivity to analog circuit errors and reduced analog processing as compared to other analog-to-digital conversion techniques. These advantages come at the expense of relatively large amount of digital processing and the working of the majorpart of circuit at a clock rate which is much higher than the analog-to-digital conversion rate. Because of using higher clock rates and feedback loop, these converters tend to be robust in the face of analog circuit imperfections [3] and do not require trimmed components which are considered necessary in conventional high precision Nyquist rate ADCs.

For these reasons, high precision $\Delta \Sigma$ ADCs can be implemented using high density VLSI processes.

$\Sigma \Delta \mathrm{ADC}$ is a system which consists of a $\Sigma \Delta$ modulator followed by a digital decimation filter. The $\Sigma \Delta$ modulator oversamples the input signal $\mathrm{i}$-e performs sampling at a rate much higher than the nyquist rate. The ratio of this rate to the Nyquist rate is called over-sampling ratio (OSR).
After over-sampling, it typically performs very coarse analog-to-digital conversion at the resulting narrow-band signal. By using coarse digital-to-analog conversion and feedback, the quantization error introduced by the coarse quantizer is spectrally shaped i-e the major portion of the noise power is shifted outside the signal band. This process is called quantization noise shaping. The digital decimation filter removes the out-of-band portion of the quantization error and brings back the output rate to Nyquist rate.

The paper is organized as follows. Section II presents a review of $\Sigma \Delta$ modulator with theoretical analysis. Section III presents the described structure of the second order $\Delta \Sigma$ modulator with simulations results. In section IV, all main parameters of the proposed modulator are indicated with a full comparison of the most popular designs in which the performance of each modulator is cited in table IV. Conclusion is drawn in Section V.

\section{REVIEW OF SIGMA-DELTA MODULATOR}

Signal-to-noise ratio (SNR) and Dynamic range (DR) are two most important specifications commonly used to characterize the performance of over-sampling sigma-delta ADCs [5]. The system design begins with the calculation of the dynamic range. According to the design theory, the DR of a sigma-delta modulator can be gotten by following formula.

$D R(d B)=10 \log _{10}\left[\frac{3}{2} \frac{(2 L+1)}{\Pi^{2 L}} \operatorname{OSR}^{2 L+1}\left(2^{B}-1\right)^{2}\right]$ (1)

The theoretical DR is a function of the modulator order L, oversampling ratio OSR, and the numbers of bits in the quantizer B.

The expression (1) reveals that an additional bit in the internal quantizer can roughly obtain a 6-dB improvement of DR. This improvement is independent of the OSR, while the improvement obtained with increasing the order $\mathrm{L}$ is dependent on it. The DR of a theoretical L th-order Sigma-Delta converter increases with OSR in $(L+1 / 2)$ bits/octave. This is shown in Figure 1, where the DR is plotted as a function of the oversampling ratio and the modulator order, in case of a singlebit internal quantizer. 


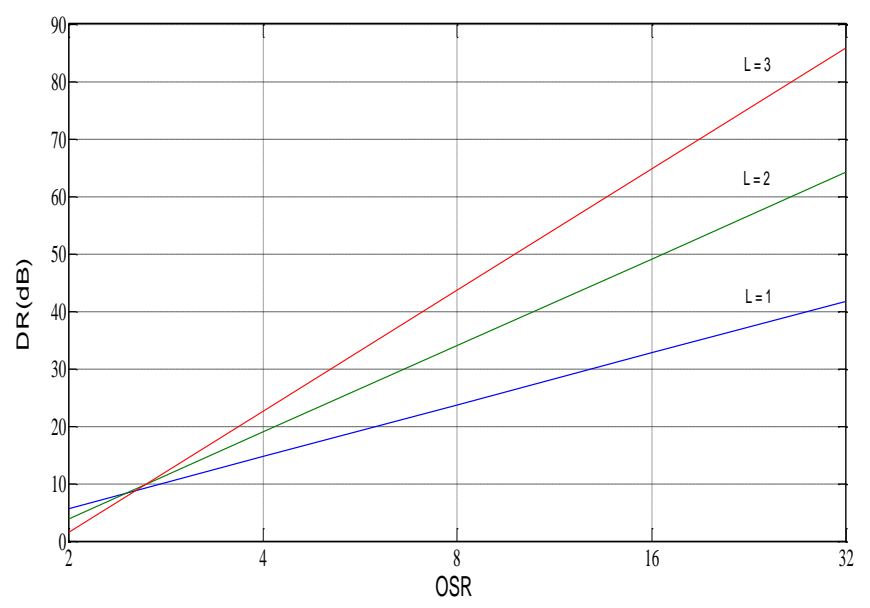

Figure 1.DR Vs OSR of Lth-order theoretical $\Sigma \Delta$ Modulators

The SNR of a converter is the ratio of the input signal power to the noise power measured at the output of the converter. The maximum SNR that a converter can achieve is called peak signal-to-noise-ratio $\left(\mathrm{SNR}_{\mathrm{p}}\right)$. The noise here should include the quantization and circuit noise. The SNRP of the Lth order Sigma-Delta modulator can be calculated as:

$$
S N R_{P}=\frac{3 \Pi}{2}\left(2^{B}-1\right)^{2}(2 L+1)\left(\frac{O S R}{\Pi}\right)^{2 L+1}(2)
$$

The SNR of the $\Sigma \Delta$ ADC can be increased by $(2 \mathrm{~L}+1) 3 \mathrm{~dB}$, or $\mathrm{L}+0,5$ bits by doubling the oversampling ratio, where $\mathrm{L}$ denotes the order of the loop filter. It is tempting to raise the oversampling ratio to increase the SNR of the Sigma-Delta modulator. However, it is restricted by the speed limit of the circuit and the power consumption.

In practice, for the same performance, it is preferred to lower the oversampling ratio. Another driving force is the ever increasing bandwidth requirement, which also needs to lower the oversampling ratio.

For high bandwidth converters, the oversampling ratio should be kept as low as possible. A lot of efforts have been made at the system level to lower the oversampling ratio and maintain the same performance.

Starting from the desired 14-bit of resolution, the SigmaDelta ADC was designed. The DR is computed as follows:

$$
\begin{aligned}
& D R^{2}=3\left(2^{2 \times N-1}\right) \\
& D R^{2}=3\left(2^{2 \times 14-1}\right)
\end{aligned}
$$

Where $\mathrm{N}$ represents number of bits, hence $\mathrm{DR}(\mathrm{dB})$ is equal to $86.04 \mathrm{~dB}$. For computing the OSR for first, second and third order devices, a small MATLAB script was developed using the following equation:
$O S R=\left(\frac{2}{3} \frac{D R^{2} \Pi^{2 L}}{2 L+1}\right)^{\frac{1}{2 L+1}}$

OSR means Over Sampling Ratio which is based on the following formula:

$O S R=\frac{F_{s}}{2 \times f_{b}}(6)$

If we choose an OSR equal to $88, \mathrm{~F}_{\mathrm{s}}$ is the sampling frequency which is calculated to $14.08 \mathrm{MHz}$, and $\mathrm{f}_{\mathrm{b}}$ means base band frequency $(80 \mathrm{KHz})$. Table I shows the results of the equation (4) for a first, second and third order.

TABLE I. OSR FOR FIRST TO THIRD MODULATOR'S ORDER

\begin{tabular}{|c|c|}
\hline Modulator's order & OSR \\
\hline $1^{\text {rst }}$ & 960 \\
\hline $2^{\text {nd }}$ & 88 \\
\hline $3^{\text {rd }}$ & 24 \\
\hline
\end{tabular}

For a first order modulator an OSR of 960 is needed, given a relatively high sampling frequency for the $0.35 \mu \mathrm{m}$ CMOS ultra-low-voltage system. For the second and third order devices, better sampling frequencies are required. Although for the third order modulator, the lowest sampling frequency is needed, this frequency implies the use of higher consumption and taking up more area. For that reason, a second order modulator was chosen for this application.

$\mathrm{N}$ is the effective number of bits of $\Delta \Sigma$ converter which is given by [6]:

$$
N=\frac{1}{2} \log _{2}\left[(2 B-1)^{2}(2 L+1) O S R^{2 L+1} /(\pi)^{2 L}\right](7)
$$

Where $\mathrm{B}$ represents number of bits of the quantization circuitry $(\mathrm{B}=1)$.

In this case, OSR is equal to $88, F_{s}$ is the sampling frequency which is calculated to $14.08 \mathrm{MHz}$. We obtained an effective number of bits equal to 14 bits which verify the two last results in equations (1) and (3).

\section{PROPOSED SECOND ORdER SIGMA -DELTA MODULATOR}

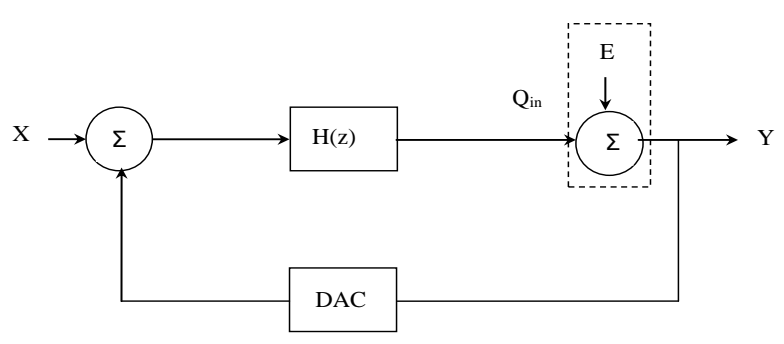

Figure 2. Linear model of conventional first order $\Sigma \Delta$ Modulator 
As shown in figure 2, first-order $\Sigma \Delta$ modulator has the advantages of being simple, robust and stable. Despite these good points, its overall performance in terms of resolution and idle-tone generation is inadequate for most applications. Second-order $\Sigma \Delta$ modulator overcomes these disadvantages at the expense of increased circuit complexity and reduced signal range. According to figure 2 , the modulator can be considered as a two-input [E (z) and $\mathrm{X}(\mathrm{z})]$, one-output $[\mathrm{Y}(\mathrm{z})]$ linear system. The output signal is expressed as:

\section{$Y(z)=\operatorname{STF}(z) \cdot X(z)+N T F(z) \cdot E(z)(8)$}

Where $\operatorname{STF}(z)$ is the signal transfer function and NTF (z) is the noise transfer functions, which are given by:

$$
\begin{aligned}
& \operatorname{STF}(z)=\frac{Y(z)}{X(z)}=\frac{H(z)}{1+H(z)}(9) \\
& \operatorname{NTF}(z)=\frac{Y(z)}{E(z)}=\frac{1}{1+H(z)}(10)
\end{aligned}
$$

By using superposition principle, the output signal is obtained as the combination of the input signal and the noise signal, with each being filtered by the corresponding transfer function:

$$
Y(z)=\operatorname{STF}(z) \cdot X(z)+N T F(z) \cdot E(z)(11)
$$

If we choose $\operatorname{STF}(\mathrm{z})$ equal to $\mathrm{Z}^{-1}$ and $\operatorname{NTF}(\mathrm{z})$ equal to $1-\mathrm{Z}^{-1}$ we obtain:

$$
\begin{aligned}
& \operatorname{STF}(z)=z^{-2}(12) \\
& \operatorname{NTF}(z)=\left(1-z^{-1}\right)^{2}(13)
\end{aligned}
$$

In this case, the output signal for the ideal linear model can be written as:

$$
Y(z)=X(z) \cdot z^{-2}+E(z) \cdot\left(1-z^{-1}\right)^{2}(14)
$$

To achieve the objective cited above, All main parameters of the described modulator are summed up in Table II.

TABLE II. DESIGNED MODULATOR PARAMETERS

\begin{tabular}{|c|c|}
\hline Parameters & Value \\
\hline Order of modulator & 2 \\
\hline Sampling Frequency (clock) & $14.08 \mathrm{MHz}$ \\
\hline Signal Band width & $80 \mathrm{KHz}$ \\
\hline Over sample Ratio(OSR) & 88 \\
\hline Resolution & 14 bits \\
\hline
\end{tabular}

The functional diagram of the second order modulator simulated using Simulink in MATLAB is shown in Figure 3. The single bit DAC is replaced by a simple wire. The input is a sinusoidal signal with $0.4 \mathrm{~V}$ amplitude and frequency $20 \mathrm{kHz}$. This signal is fed through two integrators and is connected to the comparator at the output.

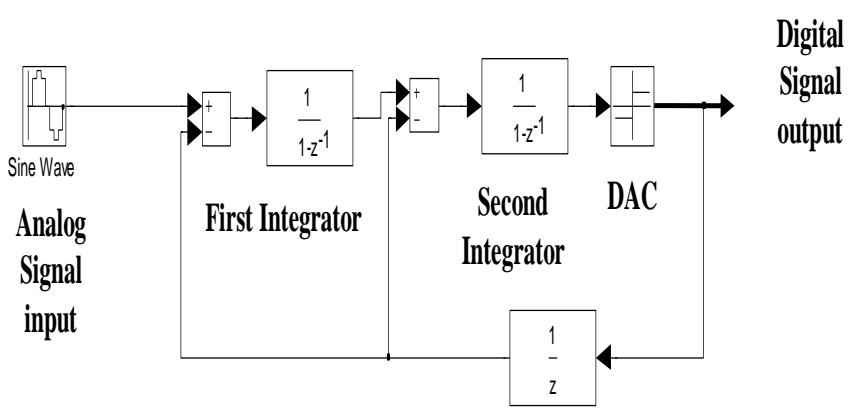

Figure 3. Block diagram of Second Order $\Sigma \Delta$ Modulator

The modulated output as seen through the scope is shown in Figure 4 with the input signal overlaid on it.

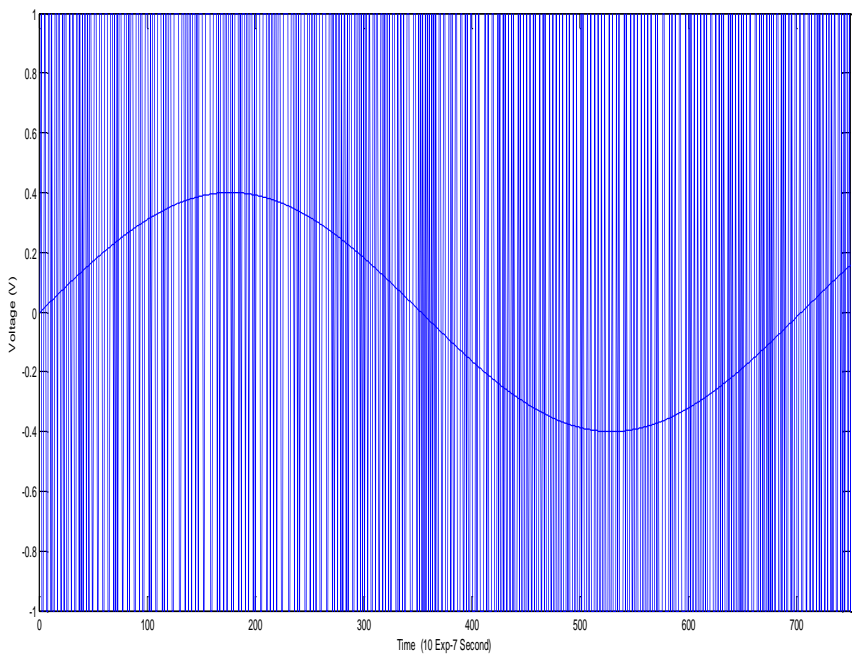

Figure 4. Pulse density output from a sigma-delta modulator for a sine wave input

A discrete Fourier Transform (DFT) of the sampled output signal is performed to calculate the SNR of the system. The logarithm of the amplitude of the signal is plotted versus the signal frequency and the SNR is found to be close to $85.17 \mathrm{~dB}$ as shown in Figure 5.

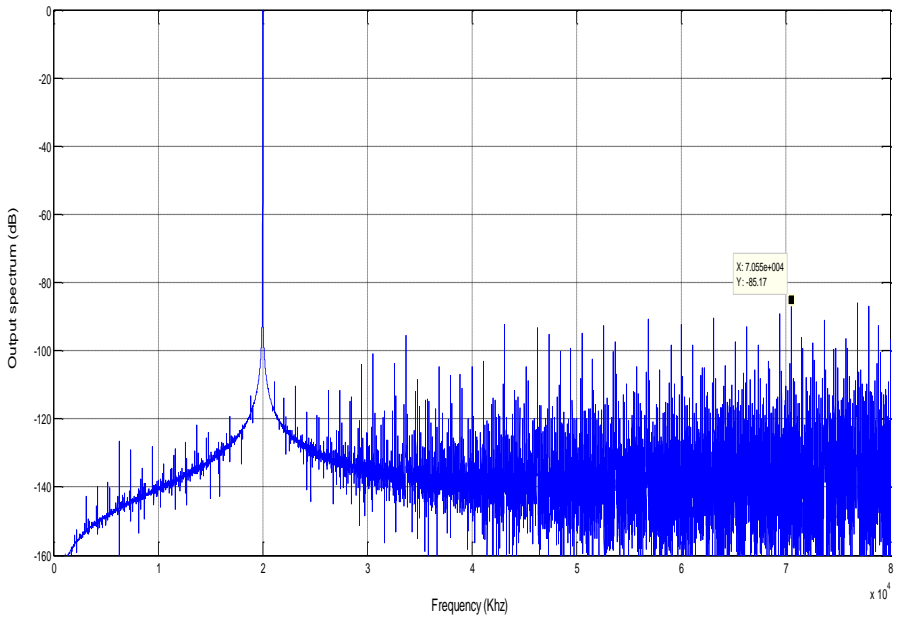

Figure 5. Frequency spectrum zoom of the modulated signal 
It can be seen that second order noise shaping is taking place wherein most of the noise is pushed to the higher frequency bands as shown in figure 6. The original signal can be retrieved using a digital low pass filter.

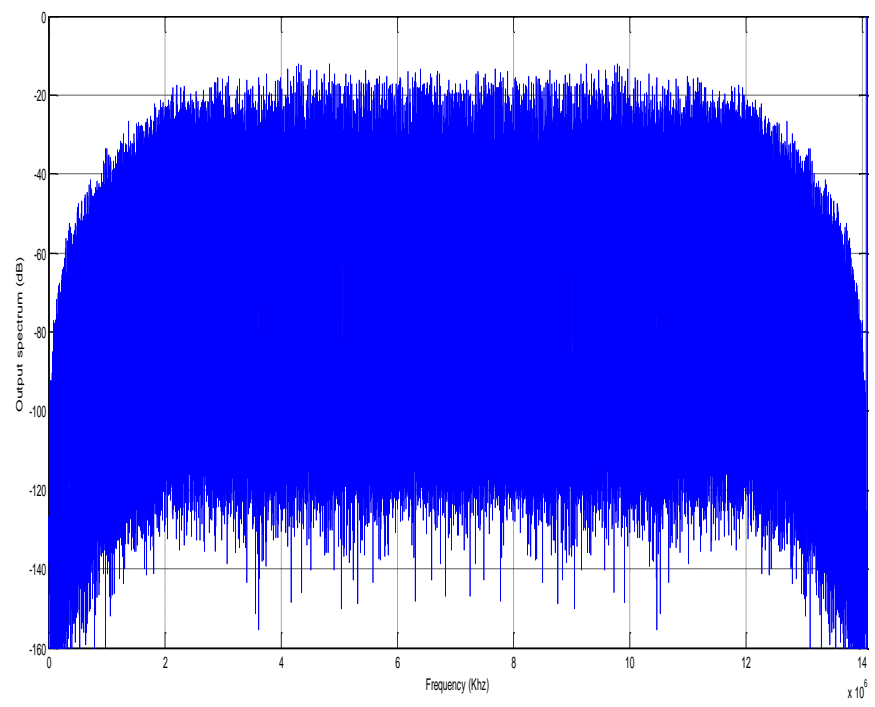

Figure 6. Frequency spectrum of the modulated signal

Figure 7 shows a block diagram of a complete Secondorder $\Sigma \Delta$ modulator. It is made up of two integrators, a comparator, and digital to analog converter (DAC). These include switches Q and Q' for applying one of two reference node voltages, $+\mathrm{V}_{\text {ref }}$ and $-\mathrm{V}_{\text {ref }}$, depending on comparator output polarity. The two integrators are based on amplifier, and use two phase, with the respective phases denoted by $\phi 1$ and $\phi 2$.

As shown in figure 7, signal sampling is completed by the first integrator connecting to the input. For this reason, the amplifier determines the whole performance of the sigma-delta modulator and needs to be carefully designed. Each integrator is made up of one operational amplifier. This operational amplifier has an adequate bandwidth and higher voltage gain. It is composed of two input transistors formed by P-channel MOSFETs M1 and M2 in order to reduce 1/f noise [7].

This stage of op-amp is also formed by N-channel MOSFETs, M3 and M4.The use of transistor M7 as a Pchannel common source amplifier forms the second stage of op-amp. The polarisation block is formed by ten transistors (M5, M6, M8, M9, M10, M11, M12, M13, M14 and M15) with variable input voltage $V_{\text {in }}$ and input resistance $R_{\text {in }}$.

The overall gain of the amplifier is found to be given by:

$$
A_{v}=g_{m 1}\left(r_{d s 2} / / r_{d s 4}\right) \cdot g_{m 7}\left(r_{d s 6} / / r_{d s 7}\right)(15)
$$

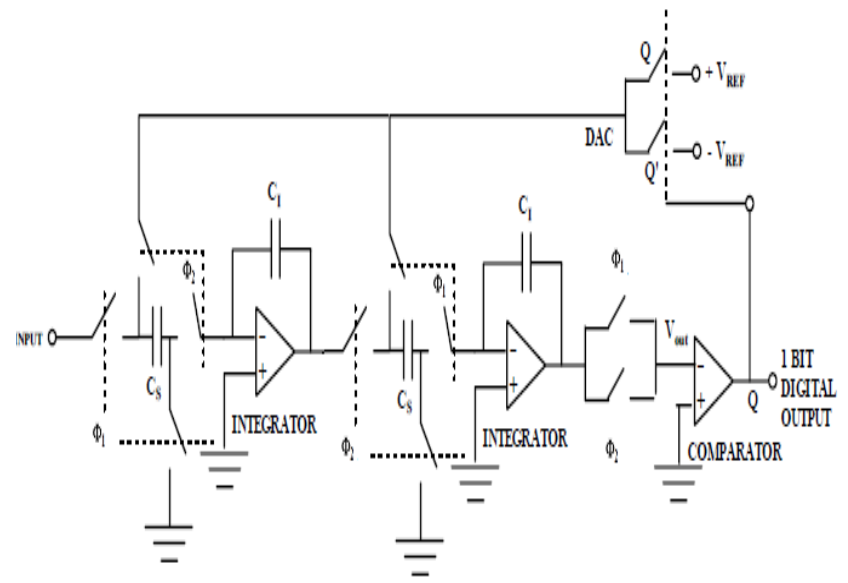

Figure 7. A complete Second-order $\Sigma \Delta$ modulator

Where $g_{m i}$ is the transconductance and $r_{d s i}$ is the drain to source resistance of $\mathrm{i}^{\text {th }}$ transistors with $\mathrm{i}=1,2,4,6,7$.

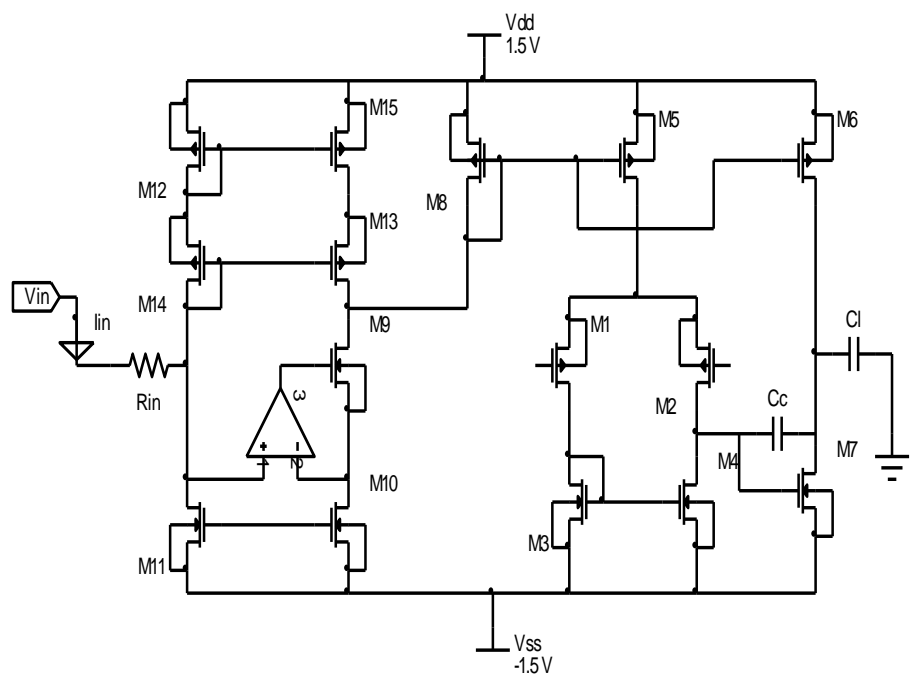

Figure 8. Miller Operational Transconductance Amplifier

Considering the appeal to moderate speed and noise of operational amplifier together with the low power consumption of the operational amplifier, Miller structure is chosen, and the structure of circuit is shown in figure 8 . The system has problems of instability because the signal will pass through two-stage circuit and may bring extra pole and zero in this operational amplifier, so it requires importing frequency compensation. Simulations results using T-spice shows that output frequency response of our operational amplifier achieves a gain of $60 \mathrm{~dB}$ with a large gain bandwidth of 82 $\mathrm{MHz}$ and a phase margin of $62^{\circ}$ to ensure more stability (figure 9). 


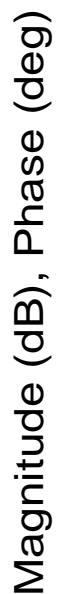

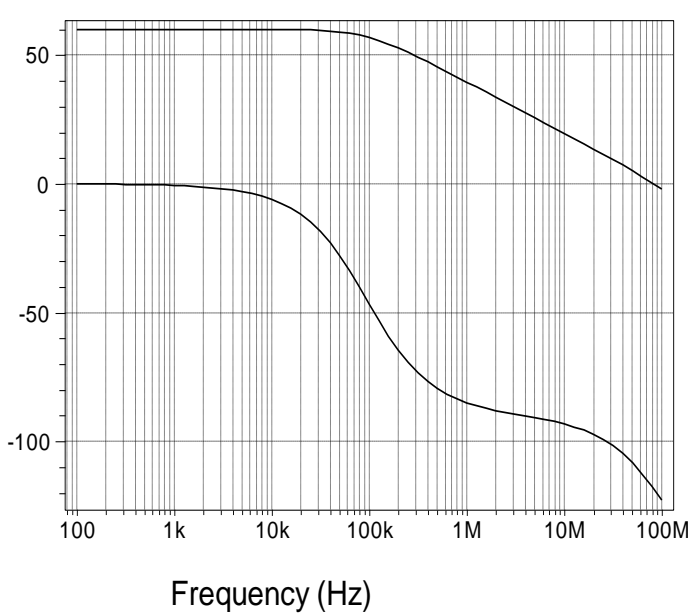

Figure 9. Frequency response of Operational Transconductance Amplifier

\section{A. Why high performance and low power?}

With the rapid development of computers and communications, a very large number of chips required to have higher performance, low power and small size. Hence analogue, mixed signals with low power and especially SigmaDelta modulators become more and more important. Operational amplifier structure for almost all modulators circuits determine the performance of analogue structure, which largely depends on their characteristics. One of the popular op-amp is a two-stage Miller op-amp. It introduces an important concept of compensation. The object of this compensation is to maintain stability of the operational amplifier. This last is made up of three stages even though it is often referred to as a "two-stage" op-amp, ignoring the buffer stage. The first stage is composed of the input devices of the differential pair which are formed by P-channel or N-channel MOSFETs. It plays a very important role due to its differential input to single-ended output conversion and its high gain. In addition this stage of op-amp also had the current mirror circuit formed by N-channel MOSFETs. In the other hand the second stage is formed by only one transistor which serves as a Pchannel common source amplifier. The current $\mathrm{I}_{\text {bias }}$ of the opamp circuit goes through current mirrors formed by the rest of P-channel transistors in order to produce a low current of $10 \mu \mathrm{A}$ [17].

According to figure 10, the DC characteristic for the V-I converter for different values of resistance $\left(\mathrm{R}_{\text {out }}=100 \Omega\right.$, $R_{\text {out }}=2.5 \mathrm{~K} \Omega, R_{\text {out }}=5 \mathrm{~K} \Omega$ ) is presented. In the one hand the full input voltage swing capability is evident with truly linearity. In the other hand, in order to ensure low and low consumption an input voltage $\mathrm{V}_{\text {in }}$ varied from $-1.1 \mathrm{~V}$ to $0 \mathrm{~V}$ to provide a current from $-10 \mu \mathrm{A}$ to $150 \mu \mathrm{A}$. In this case the current is equal to $10 \mu \mathrm{A}$ at input voltage $\mathrm{V}_{\text {in }}$ equal to $-1 \mathrm{~V}$.

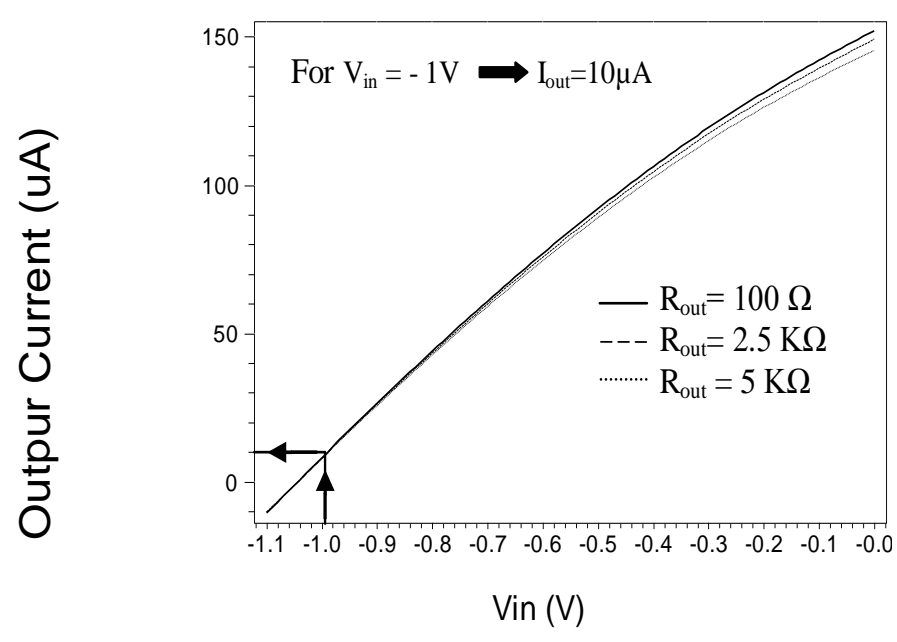

Figure 10. DC characteristics of V-I converter for different values of resistance $\mathrm{R}_{\text {out }}$

From figure 8, the Miller operational amplifier is presented. Here in the polarisation circuit, an amplifier between the mirror's input and output transistor is necessary to achieve high current copy accuracy. In the other hand we use a simple amplifier composed of only two transistors.

This amplifier is proposed by K. Tanno [19] to provide low voltage, low consumption and high performance as shown in figure 11 .
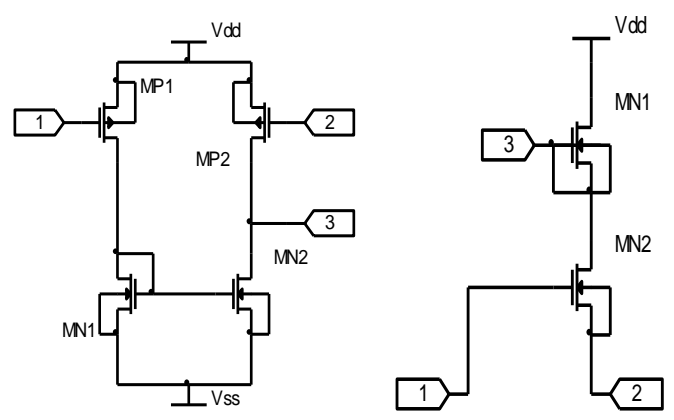

(a)

(b)

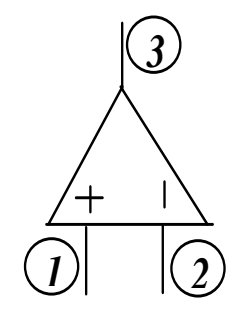

Figure 11. amplifier configuration:

(a) Simple differential amplifier structure [18]

(b)Two-transistor amplifier structure [19] 
TABLE IV. COMPARISON TABLE OF MOST POPULAR DESIGNS WITH CURRENT WORK (*)

\begin{tabular}{|c|c|c|c|c|c|c|c|c|}
\hline Resolution & OSR & $\begin{array}{c}\text { SNR } \\
(\mathbf{d B})\end{array}$ & $\begin{array}{c}\text { Speed } \\
(\mathbf{M H z})\end{array}$ & $\begin{array}{c}\text { Power } \\
(\mathbf{m W})\end{array}$ & $\begin{array}{c}\text { Process } \\
(\mathbf{C M O S})\end{array}$ & $\begin{array}{c}\text { Signal } \\
\text { Band width }\end{array}$ & $\begin{array}{c}\text { Order } \\
\text { Ref. } \\
\text { No. }\end{array}$ \\
\hline 14-bit & 166 & - & 5.312 & 0.5 & $0.35 \mu \mathrm{m}$ & $16 \mathrm{KHz}$ & 2 & 8 \\
\hline 15-bit & 16 & 96 & 40 & 44 & $0.25 \mu \mathrm{m}$ & $1.25 \mathrm{MHz}$ & 2 & 9 \\
\hline 10-bit & 128 & 68 & 1 & 0.4 & $0.18 \mu \mathrm{m}$ & $1 \mathrm{MHz}$ & 2 & 10 \\
\hline 16-bit & 64 & 93 & 3.2 & 5 & $0.35 \mu \mathrm{m}$ & $25 \mathrm{KHz}$ & 4 & 11 \\
\hline 14-bit & 24 & 85 & 2.2 & 200 & $0.35 \mu \mathrm{m}$ & $100 \mathrm{KHz}$ & 6 & 12 \\
\hline 11-bit & 10 & 62.5 & 300 & 70 & $0.13 \mu \mathrm{m}$ & $15 \mathrm{MHz}$ & 4 & 13 \\
\hline 14-bit & 96 & 85 & 53 & 15 & $0.18 \mu \mathrm{m}$ & $300 \mathrm{KHz}$ & 2 & 14 \\
\hline 16-bit & 128 & - & 5.12 & 2.6 & $0.18 \mu \mathrm{m}$ & $20 \mathrm{KHz}$ & 3 & 15 \\
\hline 8-bit & 64 & 49.7 & 1.024 & 6.6 & $0.6 \mu \mathrm{m}$ & $8 \mathrm{KHz}$ & 1 & 16 \\
\hline 14-bit* & 88 & 85 & 14.08 & 9.8 & $0.35 \mu \mathrm{m}$ & $80 \mathrm{KHz}$ & 2 & This work \\
\hline
\end{tabular}

\section{RESULTS AND COMPARISON}

The second order modulator is designed by using AMS technology 0.35 um CMOS process, the over sampling ratio is 88 with a signal band width of about $80 \mathrm{kHz}$.

All main parameters of the described modulator are summed up in Table III.

TABLE III. T DESIGNED MODULATOR PARAMETERS

\begin{tabular}{|c|c|}
\hline Parameters & Value \\
\hline Technology & AMS 0.35 $\mu \mathrm{m}$ \\
\hline Order of modulator & 2 \\
\hline Sampling Frequency (clock) & $14.08 \mathrm{MHz}$ \\
\hline Signal Band width & $80 \mathrm{KHz}$ \\
\hline Over sample Ratio(OSR) & 88 \\
\hline References & $\pm 1 \mathrm{~V}$ \\
\hline Maximum Input & $1 \mathrm{Vpp}$ \\
\hline Supply voltage & $\pm 1.5 \mathrm{~V}$ \\
\hline Resolution & $14-\mathrm{bit}$ \\
\hline Signal to Noise Ratio (SNR) & $85 \mathrm{~dB}$ \\
\hline Dynamic range (DR) & $86 \mathrm{~dB}$ \\
\hline Quantizer resolution & $1 \mathrm{bit}$ \\
\hline Power consumption & $\mathbf{9 . 8} \mathbf{~ m W}$ \\
\hline
\end{tabular}

The current state of the art in the design of $\Sigma \Delta$ modulator is limited by the technology and the sampling speeds it is able to achieve. Here is a comparison in table IV of the most popular designs which also compares the published works with the current work. It can be seen that the current work consumes less power than most published work and achieves the resolution of 14 bits using one of the technology AMS $0.35 \mu \mathrm{m}$ CMOS process.

\section{CONCLUSION}

The low-power-consumption modulator is designed with switched-capacitor techniques, and the resolution reaches 14 bits in AMS $0.35 \mu \mathrm{m}$ CMOS process. Compared to other $\Sigma \Delta$ modulators, the second order single $\Sigma \Delta$ modulator has many advantages on performance, stability, area and system specification requirements, especially the power consumption.

When the power supply is $\pm 1.5 \mathrm{~V}$, the power consumption is only $9.8 \mathrm{~mW}$.

In the future work, we will study and design of the different blocks constituting complete analogue to digital converter
(ADC) in transistor level, which is composed of an analog Sigma Delta modulator with a digital filter, in the other hand we will discuss about multi-bit discrete-time Sigma-Delta ADC.

\section{REFERENCES}

[1] Delta Sigma Data Converters: Theory, Design and Simulation. IEEE Press, IEEE Circuits and Systems Society, 1997.

[2] B. E. Boser and B. A. Wooley. The design of sigma-delta modulation and analog-to-digital converters. IEEE Journal of Solid-State Circuits, 23:1298-1308, December 1988.

[3] Oversampling Delta-Sigma Converters. IEEE Press, 1992.

[4] Top-Down Design of High-Performance Sigma-Delta Modulators. Kluwer Academic Publishers, 1999

[5] Vineeta Upadhyay and Aditi Patwa, "Design Of First Order And Second Order Sigma Delta Analog To Digital Converter",International Journal of Advances in Engineering \& Technology, July 2012.

[6] Medeiro F., del Rio R., de la Rosa J.M., Pérez-Verdù B., A Sigma-Delta modulator design exemple : from specs to measurements, Baecelonea, May 6-10, 2002

[7] David Johns and Kenneth W. Martin : "Analog Integrated Circuit Design" John Wiley \& Sons, 1997.

[8] F. Munoz, A. P. VegaLeal, R. G. Carvajal, A. Torralba, J. Tombs, J. Ramirez-Angulo, $\backslash \mathrm{A}$ 1.1V Low-Power __ Modulator For 14-b 16KHz A/D Conversion"; The 2001 IEEE International Symposium on Circuits and Systems, Vol. 1, 6-9 May 2001

[9] KiYoung Nam, Sang-Min Lee, David K. Su, and Bruce A. Wooley : " Voltage Low-Power Sigma-Delta Modulator for Broadband Analog-toDigital Conversion" IEEE JOURNAL OF SOLID-STATE CIRCUITS, VOL. 40, NO. 9, SEPTEMBER 2005

[10] H. Lee, C. Hsu, S. Huang, Y. Shih, C. Luo : " Designing low power of Sigma Delta Modulator for Biomedical Application", Biomedical Engineering-Applications, Basis \& Communications, Vol. 17 No. 4, August 2005.

[11] Hsin-Liang Chen, Yi-Sheng Lee, and Jen-Shiun Chiang: "Low Power Sigma Delta Modulator with Dynamic Biasing for Audio applications", Circuits and Systems, 5-8 Aug 2007. MWSCAS 2007. 50th Midwest Symposium on

[12] Morizio J, Hoke I M, Kocak T, Geddie C, Hughes C, Perry J,MadhavapeddiS, Hood M, Lynch G, Kondoh H, Kumamoto T, Okuda T,Noda H, Ishiwaki M, Miki T, Nakaya M, "14-bit 2.2-MS/s sigma-delta ADC's",Solid-State Circuits, IEEE Journal of, Volume 35, Issue 7, July 2000 Page(s):968 - 976.

[13] Di Giandomenico A, Paton S, Wiesbauer A, Hernandez L, Potscher T, Dorrer L, "A $15 \mathrm{MHz}$ bandwidth sigma-delta ADC with 11 bits of resolution in $0.13 / \mathrm{spl} \mu \mathrm{m}$ CMOS", Solid-State Circuits, IEEE Journal of, Volume 39, Issue 7,July 2004, Page(s): 1056- 1063. 
[14] Gaggl R, Wiesbauer A, Fritz G, Schranz Ch., Pessl P, “A 85-dB Dynamic Range Multibit Delta-Sigma ADC for ADSL-CO Applications in $0.18 \mu \mathrm{m}$ CMOS", Solid-State Circuits, IEEE Journal of, Volume 38, Issue 7, July 2003,Page(s): 1105 - 1115

[15] Chen Yueyang, Zhong Shun'an, Dang Hua, "Design of A low-powerconsumption and high-performance sigma-delta modulator", 2009 World Congress on Computer Science and Information Engineering

[16] Boujelben S, Rebai Ch., Dallet D, Marchegay Ph., "Design and implementation of an audio analog to digital converter using oversamplingtechniques". 2001 IEEE
[17] Laajimi Radwene; Gueddah Nawfil; Masmoudi Mohamed: "Low Power Variable Gain Amplifier with Bandwidth Of 80-300 MHz Using For Sigma-Delta Analogue to Digital Converter in Wireless Sensor Receiver" International Journal of Computer Applications;Apr2012, Vol. 43

[18] Ahmed Nader Mohieldin, Edgar Sánchez-Sinencio, and José SilvaMartínez : 'Nonlinear effects in pseudo differential OTAs with CMFB', IEEE Transactions On Circuits and Systems-II: Analog and Digital Signal Processing, Vol. 50, No. 10, October 2003.

[19] K. tanno, O. Ishizuka and Z. Tang : ' Low voltage and low frequency current mirror using a two- MOS subthreshold op-amp', Electronics Letters 28th March 1996 Vol. 32 No. 7 